Université du Québéc à Rimouski (Canada) Josianne Caron
mouski (Canada)

\title{
La gestion de classe au primaire en contexte de pandémie
}

Centre de services scolaire Des Samares (Canada)

Mélanie Arbour

Centre de services scolaire Des Affluents (Canada)

Tristan Brochu

Centre de services scolaire Des Affluents (Canada)

Classroom management in elementary school during the pandemic

doi: $10.18162 / f p .2020 .674$

\section{ésumé}

La gestion de classe optimale est certes tributaire d'un déploiement assuré de compétences professionnelles par l'enseignant (Gaudreau, 2017), de même que d'un contexte éducatif favorable à l'établissement et au maintien d'un climat d'apprentissage positif et sécurisant. Ce contexte se retrouve bouleversé par l'actuelle pandémie. Comment peut-on gérer sa classe lorsque plusieurs élèves sont scolarisés à distance et que d'autres sont à l'école? Cet article pose un regard sur la mise en place de pratiques adaptées relatives à la gestion de classe de quatre enseignants du primaire, et ce, à l'aide de référents théoriques et expérientiels.

Mots-clés

Gestion de classe au primaire; pratiques de gestion de classe; climat d'apprentissage; pandémie; COVID-19

\section{Abstract}

Optimal classroom management requires a surefooted use of professional teaching competencies (Gaudreau, 2017) combined with an educational context that establishes and maintains a safe and positive learning environment. This context has been upended by the current pandemic. How can teachers manage their classrooms when many students are learning remotely while others remain in school? With reference to the theoretical and experiential literature, this article examines how four elementary school teachers adapted their classroom management practices.

\section{Keywords}

Classroom management in elementary school, classroom management practices, learning environment, pandemic, COVID-19

\section{Introduction}

La gestion de classe requiert la mobilisation optimale de plusieurs compétences professionnelles attendues des enseignants. Lorsque ceux-ci déploient des pratiques relatives à la gestion de classe, ils contribuent à influencer positivement la réussite éducative des élèves sous leur responsabilité (Gaudreau, 2017). Or, on ne peut parler de gestion de classe sans parler d'organisation. Dans la situation actuelle, les enseignants font face à une désorganisation et ils doivent ainsi adapter leur gestion de classe. Nous avons voulu comprendre comment il est possible de gérer la classe lorsque plusieurs élèves sont scolarisés à distance et que d'autres le sont en présentiel, mais selon une organisation adaptée en fonction des consignes de la Direction de la santé publique (DSP). Pour ce faire, nous portons un regard sur la mise en place de pratiques adaptées relatives à la gestion de classe de quatre enseignants du préscolaire primaire. Cet article présente d'abord le contexte et la problématique à l'étude pour ensuite aborder les référents théoriques et la démarche de collecte d'informations ainsi que les référents expérientiels issus de la mise en place de pratiques relatives à la gestion de classe en contexte de pandémie.

\section{Contexte et problématique}

Chaque année, les actions visant à créer les bases d'un climat d'apprentissage positif, sain et sécurisant se mettent en place avant la rentrée scolaire et elles évoluent en cours de route (Caron, 2012). La gestion de classe est influencée par des siècles de traditions qui se perpétuent. Dans le secteur du préscolaire primaire, on s'attend à voir des élèves regroupés selon un nombre relativement normé dans un local attitré et sous la responsabilité principale d'un enseignant 
titulaire. En mars 2020, lors de l'annonce de la fermeture des écoles en raison de la pandémie occasionnée par la propagation de la COVID-19, des traditions scolaires tombaient pour une durée indéterminée, la classe se retrouvant alors complètement désorganisée. Dès lors, les enseignants ont eu à relever des défis dont la gestion de classe entièrement à distance ou, dans certaines régions, la gestion de classe comodale auprès d'élèves à distance et en présentiel.

Ce défi actuel est de taille, considérant que les enseignants sont déjà très préoccupés par des problématiques liées à la gestion de classe (Martin, Pidoux et Brülhart, 2019), notamment la complexité de l'enseignement à des élèves dont les besoins sont diversifiés (Dufour, Portelance, Pellerin et Boies, 2019 ; Leroux et Paré, 2016). Par ailleurs, toute période de changement, de précarité ou d'instabilité professionnelle vient entraver la capacité à gérer la classe (Harvey, à paraître). L'instabilité professionnelle complexifie plus précisément la relation maître-élèves, un des facteurs primordiaux et nécessaires à l'établissement et au maintien d'une bonne gestion de classe (Martineau et Vallerand, 2007).

Nombreux sont les enseignants éprouvant le besoin de développer leur compétence et leur sentiment d'efficacité personnelle relativement à la planification, l'organisation et la supervision du mode de fonctionnement du groupe-classe en vue de favoriser l'apprentissage et la socialisation des élèves (Girouard-Gagné et Paré, 2015 ; Martin, Pidoux et Brülhart, 2019). Comme l'établissement et le maintien d'un climat d'apprentissage s'avèrent difficiles et complexes lorsque l'enseignant voit ses repères s'effriter (Duchesne et Kane, 2010), il apparaît pertinent de poser un regard sur le contexte de la pandémie dans une visée de développement professionnel. Comment des enseignants ont-ils adapté leur gestion de classe? Avec quelles pratiques et selon quelles intentions? Cet article vise à documenter, à l'aide de référents théoriques inspirés des cinq composantes de la gestion de classe (Gaudreau,2017) et des référents expérientiels, la mise en place de pratiques de gestion de classe susceptibles d'établir et de maintenir un climat d'apprentissage chez des élèves en contexte de pandémie. Ces voies d'exploration pourraient aider des enseignants, voire des novices et des futurs enseignants, à atténuer le choc de la confrontation avec la réalité du quotidien en contexte de pandémie.

\section{Référents théoriques}

Les référents théoriques qui ont servi à analyser les informations collectées sont présentés dans les paragraphes suivants. En premier lieu, le concept de gestion de classe est abordé. Sont précisées ensuite cinq composantes essentielles à la gestion de classe.

La gestion de classe se conçoit comme «[...] l'ensemble des actes réfléchis, séquentiels et simultanés que le personnel enseignant conçoit, organise et réalise pour et avec les élèves dans le but d'établir, maintenir ou restaurer un climat favorisant l'engagement des élèves dans leurs apprentissages et le développement de leurs compétences» (Gaudreau, 2017, p. 7). Caron, Portelance et Marzouk (2020) ajoutent que l'établissement de ce climat vise à prévenir ou anticiper des situations problématiques et que, lorsque la restauration du climat de classe s'avère nécessaire, la résolution de problèmes devrait idéalement être planifiée et réalisée en collaboration interprofessionnelle et intersectorielle. La collaboration réfère à une démarche conjointe d'acteurs concernés par les différents besoins des élèves.

2 - Formation et profession 28(4 hors-série), 2020 
Pour outiller les enseignants et leurs collaborateurs de manière à ce qu'ils puissent mettre en ouvre des pratiques de gestion de classe optimales, Gaudreau (2017) suggère cinq composantes inhérentes à celle-ci : la gestion des ressources ; l'établissement d'attentes claires; le développement de relations sociales positives ; l'attention et l'engagement des élèves sur l'objet d'apprentissage et la gestion des comportements d'indiscipline.

\section{La gestion des ressources}

La gestion des ressources concerne cinq aspects: le temps, l'espace, le matériel, les ressources technologiques et les ressources humaines. Chacun d'eux doit être pris en considération en vue de structurer l'enseignement. Lorsque les aspects sont réfléchis et mobilisés en symbiose, ils permettent d'établir une bonne gestion de classe. La gestion des ressources débute par une planification judicieuse des activités d'enseignement-apprentissage suivie d'une animation de celles-ci.

\section{L'établissement d'attentes claires}

L'établissement d'attentes claires porte sur la mise en place de règles de conduite et de routines, la formulation de directives précises et l'enseignement de procédures. Lorsqu'elles sont connues, les attentes permettent aux élèves de savoir ce qui est attendu d'eux. Les consignes leur sont transmises à l'aide de directives alpha, lesquelles se composent d'un contact visuel, d'une consigne courte et précise, d'une mention d'un comportement observable attendu, d'une formulation à la forme déclarative ou impérative. Elles sont enseignées de manière explicite. Lorsque l'enseignant observe un respect des attentes, il utilise la rétroaction positive multiforme pour encourager les élèves à adopter et maintenir les comportements attendus.

\section{Le développement de relations sociales positives}

L'acte pédagogique met principalement en interaction un enseignant et des apprenants. Pour être sain et sécurisant, le lien significatif qui se dégage de cette interaction est rendu possible grâce au développement de relations interpersonnelles basées sur des valeurs et des croyances dites prosociales, et ce, entre l'enseignant et les élèves, entre les élèves et entre les parents et l'enseignant. Le respect mutuel, l'acceptation de l'unicité, la cohésion, la complémentarité et la réciprocité sont sous-jacents aux relations sociales positives. Dans son rôle, l'enseignant représente un agent de socialisation qui valorise le développement d'habiletés sociales et communicationnelles.

\section{L'attention et l'engagement des élèves sur l'objet d'apprentissage}

L'attention et l'engagement des élèves se caractérisent par une motivation à apprendre et à se comporter en fonction des attentes à leur égard. Cette motivation est tributaire d'une perception positive de la tâche, d'une participation active des élèves par une mobilisation cognitive et comportementale, mais aussi de la personnalité de l'enseignant et de ses efforts pour rendre intéressants, voire captivants, les objets d'apprentissage. Les stratégies adoptées par les enseignants pour motiver les élèves sont variées et différenciées. Selon les besoins de ceux-ci, les contenus, les structures, les processus et les productions peuvent être flexibles, adaptés ou modifiés. Pour chaque élève, le maintien de l'attention et de l'engagement est facilité par la supervision personnalisée de la progression des apprentissages. 


\section{La gestion des comportements d'indiscipline}

La gestion des comportements d'indiscipline réfère à la nécessité d'intervenir en présence de distraction, de désengagement, de désobéissance et de manifestations comportementales à dominance extériorisée ou intériorisée. L'élève qui choisit de manifester des comportements inadéquats amène l'enseignant à user de plusieurs stratégies d'intervention. Les stratégies sont dites préventives lorsqu'elles consistent à enseigner explicitement des comportements attendus; fournir une aide opportune; exploiter la proximité, la guidance physique et le toucher; limiter l'espace et le matériel; évaluer le comportement; anticiper les difficultés; collaborer avec les acteurs à l'intérieur et hors de l'école. Elles sont dites réactives lorsqu'elles consistent à renforcer les comportements attendus; adopter l'ignorance intentionnelle des comportements inadéquats; formuler une demande verbale; exploiter le non verbal ou retirer l'élève.

\section{Démarche de cueillette d'informations et présentation des enseignants}

Pour atteindre l'objectif de l'étude, des informations ont été recueillies auprès de quatre enseignants du préscolaire primaire ayant accepté de collaborer à l'écriture de cet article. Lors d'entretiens individuels non directifs et libres entre juin et juillet 2020, ils ont témoigné de la mise en place de pratiques adaptées relatives à la gestion de classe en contexte de pandémie, et ce, en fonction des cinq composantes de la gestion de classe (Gaudreau, 2017). À l'occasion, ils ont répondu par clavardage, à des questions visant à préciser leur témoignage.

Les propos écrits et oraux des collaborateurs ont été codifiés selon les cinq catégories préétablies. Les quatre enseignants ont également participé à l'écriture de l'article à titre de coauteurs. À partir d'un premier jet, ils ont proposé des modifications. Cette écriture collaborative a permis de se rapprocher du sens que ces praticiens donnent à leur agir et de tenir compte fidèlement du contexte de leur pratique professionnelle (Savoie-Zajc, 2019). Les quatre enseignants collaborateurs sont présentés dans le tableau 1.

Tableau 1

Caractéristiques des enseignants et de leur contexte d'enseignement

\begin{tabular}{|c|c|c|c|c|}
\hline Nom & Tristan & Mélanie & Stéphanie & Sandra \\
\hline $\begin{array}{l}\text { Années d'expérience en enseigne- } \\
\text { ment }\end{array}$ & 13 ans & 14 ans & 13 ans & 11 ans \\
\hline Centre de services scolaire & Des Affluents & Des Affluents & Des Samares & Des Navigateurs \\
\hline Niveau scolaire & $4^{e}$ année & Préscolaire & $\begin{array}{l}6 \text { e année } \\
\text { ( } 2 \text { groupes en } \\
\text { coenseigement) }\end{array}$ & $3^{e}$ année \\
\hline Nombre d'élèves & 17 élèves & 18 élèves & 39 élèves & 23 élèves \\
\hline Indice de milieu socio-économique & 9 & 4 & 10 & 1 \\
\hline $\begin{array}{l}\text { Mode d'enseignement en contexte } \\
\text { pandémique }\end{array}$ & $\begin{array}{l}\text { Exclusivement à } \\
\text { distance }\end{array}$ & $\begin{array}{l}\text { Exclusivement à } \\
\text { distance }\end{array}$ & $\begin{array}{l}\text { Exclusivement à } \\
\text { distance + quelques } \\
\text { visites en classe pour } \\
\text { maintenir le lien avec } \\
\text { la coenseignante et ses } \\
\text { élèves en présence }\end{array}$ & $\begin{array}{l}\text { Comodal (à } \\
\text { distance et en } \\
\text { présentiel) }\end{array}$ \\
\hline
\end{tabular}




\section{Référents expérientiels}

En fonction des cinq composantes présentées dans les référents théoriques, un regard est porté sur la mise en place de pratiques et intentions relatives à la gestion de classe en contexte de pandémie

\section{La gestion repensée des ressources}

Avant la fermeture des écoles visant à limiter la propagation de la COVID-19, les enseignants interrogés avaient eu la chance d'accueillir chaleureusement leurs élèves lors de la rentrée scolaire et de vivre une bonne partie de celle-ci en mode présentiel. Ils s'entendent pour dire que la réponse des élèves à leurs demandes était déjà bonne, mais qu'elle a été fragilisée par l'annonce du confinement. Dès le début de la crise actuelle, les enseignants ont alors eu à repenser la gestion des ressources.

Les enseignants ont eu deux semaines pour repenser les ressources en fonction d'une formation à distance inattendue. Dans un premier temps, il leur a fallu inviter les familles à répondre à un court sondage (téléphonique ou en ligne). Ce dernier visait à identifier le matériel informatique auquel elles avaient accès et les conditions dans lesquelles elles pouvaient permettre aux enfants d'être scolarisés à distance. Les données colligées ont été nécessaires pour prévoir le prêt de tablettes, de portables et la récupération de matériel. Stéphanie apporte cette précision : «Dans mon école, le prêt des outils techno s'est fait à l'annonce de la réouverture des écoles grâce à l'initiative de l'équipeécole. Avant cela, nous devions composer avec le peu d'outils pour plusieurs enfants dans une même famille. Il fallait ajuster les horaires des vidéoconférences en fonction de la réalité des familles ».

Une réorganisation du temps d'apprentissage avec le soutien de l'équipe-école, des conseillers technopédagogiques et d'un cours en ligne sur la formation à distance s'est également avérée nécessaire. Les enseignants transmettaient une planification hebdomadaire facultative à l'adresse courriel de tous les parents, sur la plateforme numérique de la classe, sur le portfolio numérique de l'enfant ou à l'aide d'un outil de clavardage avec les parents. Il était certes crucial d'établir avec les parents un moyen de communication efficace. Tristan relate ceci : «Des parents pouvaient nous reprocher de ne pas avoir reçu la planification. En réalité, un parent pouvait avoir changé d'adresse courriel et je nen étais pas avisé ». Chaque envoi avait pour but d'informer les élèves des tâches à effectuer, du temps dont ils disposent pour la réalisation ainsi que de la façon de déposer les extrants afin d'obtenir une rétroaction. Ces planifications s'inspiraient notamment des trousses pédagogiques du ministère de l'Éducation et de l'Enseignement supérieur (MÉES) jointes à chaque envoi. Concernant les trousses, les quatre enseignants interrogés y mettaient tous leur touche personnelle et y ajoutaient un horaire de rencontres synchrones à raison d'une à trois rencontres par semaine au début du confinement. L'annonce du retour en classe pour certains élèves et dans certaines régions a eu pour effet d'intensifier le nombre et la durée des rencontres synchrones. D'une durée moyenne de 30 minutes à 1 heure par rencontre, les enseignants s'entretenaient alors avec les élèves entre 7 à 12 fois par semaine. Ils communiquaient plus fréquemment avec les élèves en difficulté.

Pour tous les enseignants interrogés, le défi de la gestion de l'espace est apparu rapidement. Ayant l'habitude d'utiliser des espaces d'affichage pour mettre en évidence des informations importantes et significatives, les enseignants se sont tournés vers des alternatives. Pour l'enseignement à distance, les plateformes numériques de classe (ex. : Google Classroom), de type portfolio (ex. : Seesaw, Dojo) ou 
les outils collaboratifs de type mur virtuel (ex. : Padlet) ont servi à afficher des règles de classe, des référentiels (aide-mémoire, procédurier), voire à recueillir des travaux. Le matériel de manipulation et les ateliers d'apprentissage n'étaient plus accessibles en classe pour Mélanie, enseignante dans une classe préscolaire près de l'épicentre de la COVID-19 qui n'a pu ouvrir ses portes. Elle déclare : «Je lançais des défis à mes élèves comme de créer une suite logique ou de trouver des objets commençant par le son b à partir des objets du quotidien. Lors des rencontres synchrones, ils faisaient une présentation. Ils préparaient un bac d'objets, on manipulait ensemble à l'écran pour le dénombrement et autres concepts mathématiques".

Au moment du retour à l'école de mi-mai, Sandra a renoncé à contrecœur à sa classe flexible. Son aménagement était au service de la pédagogie qui l'habite et des modèles d'enseignement qui lui sont chers, dont l'apprentissage coopératif concrétisé par des îlots de coéquipiers. Elle a tout de même su imaginer des options permettant l'apprentissage coopératif à distance, dans le respect des mesures de distanciation physique. Les bureaux étaient placés selon les traditionnels « rangs d'oignons » et le sol exploité pour « répondre à trois nécessités : favoriser plusieurs formes de coopération, autoriser quelques déplacements (pour rencontrer un camarade ou l'enseignant) et réduire les croisements entre enfants " (Connac, 2020, paragr.4). En classe, le travail en groupe tenant compte des directives de la DSP a été rendu possible. Par exemple, Sandra pouvait proposer un problème mathématique à raisonner en équipe. Pour ses élèves à distance, ils pouvaient participer aux échanges, faire émerger des désaccords, en étant connectés dans la salle de vidéoconférence (Zoom) et visibles au tableau numérique interactif.

De plus, le contexte de la pandémie a motivé Sandra à expérimenter la classe extérieure. Avec les élèves assis en tailleur, à deux mètres l'un de l'autre, sous les arbres de la cour, elle a notamment enseigné la notion de l'arrondissement en apportant avec elle un tableau blanc effaçable ainsi que les manuels des élèves. Elle se remémore quelques souvenirs positifs : « La classe extérieure, c’était notre coup de cœur de la semaine. Malgré quelques rafales de vent, c'était très agréable ! Une journée ensoleillée, nous avons lu l'album $A u x$ toilettes', les élèves devaient inventer un petit sketch en lien avec le livre. Une autre classe verte a été de sortir en forêt pour faire un cherche et trouve en équipe. Encore une fois, on a eu beaucoup de plaisir au grand air ». L'enseignante manifeste un certain intérêt à exploiter à nouveau la classe extérieure. Cela peut rejoindre l'élan actuel pour l'intervention éducative, voire psychosociale par la nature et l'aventure (Rojo et Bergeron, 2017).

\section{L'établissement de nouvelles attentes claires}

Dans le contexte pandémique, les élèves devaient tenir compte de nouvelles règles à respecter. L'établissement de nouvelles attentes à l'égard des élèves a contribué à optimiser la gestion de classe, mais également à protéger la santé publique.

À distance, les enseignants ont expliqué ce qu'est le coronavirus ainsi que les mesures sanitaires en vigueur, et ce, dans un langage clair. Pour le préscolaire, Mélanie a utilisé les affiches explicatives de l'auteure Élise Gravel ${ }^{2}$ : «L'auteure propose des affiches sous forme de bandes dessinées pour expliquer : le coronavirus, le lavage des mains, l'installation du masque en respectant les mesures d'hygiène et la reconnaissance des fausses nouvelles. J'avais mes élèves à distance, mais j'ai pris au sérieux mon rôle d'éducation aux nouvelles règles pour la santé publique ».

6 - Formation et profession 28(4 hors-série), 2020 
Pour leur enseignement à distance, Stéphanie, Mélanie et Tristan ont interrogé par vidéoconférence les élèves sur les comportements qu'ils estiment nécessaires à l'établissement d'un climat sain et favorable à leurs apprentissages. En fonction de ces échanges, ces enseignants ont rapidement abordé la nétiquette avec les élèves et les parents qui les encadraient à l'écran, soit un ensemble de règles de savoir-vivre pour l'apprenant en visioconférence. Tristan insiste sur l'importance de celles-ci pour éviter des situations problématiques : «À l'écran, on pouvait voir des membres de la famille des élèves portant des vêtements inappropriés, des bagarres au sein de la fratrie. On pouvait entendre des chiens aboyer et j’en passe ». Quant à elle, Stéphanie a partagé avec ses élèves des vidéos de sensibilisation à la nétiquette. Dans les capsules vidéo qu'elle a créées, elle formulait les quatre règles principales et elle nommait les comportements attendus pour chacune de manière positive, autant que possible, et les modélisait. D'autres enseignants dans son entourage privilégiaient des infographies portant sur la nétiquette, notamment celle produite par le Service national du récit de la Formation à distance ${ }^{3}$.

Pour sa part, Sandra a vécu la rentrée de mai, comme elle l'appelle. En cohérence avec les règles émises par la DSP, des attentes touchant la sphère de la socialisation responsable devaient désormais s'inviter dans l'école : le lavage des mains, la distanciation physique, l'étiquette respiratoire, par exemple. C'est toute l'équipe-école qui a été mise à contribution pour clarifier de nouvelles attentes en vue de préparer une partie des élèves à revenir à l'école. Les membres de l'équipe-école ont notamment enseigné explicitement trois nouvelles procédures relatives à la circulation dans l'école lors de l'entrée et de la sortie, aux récréations et aux toilettes. La direction d'école et son équipe a utilisé un réseau social ${ }^{4}$ pour y publier des vidéos explicatives. Les parents recevaient un courriel invitant à visionner, avec leur enfant, ces capsules vidéo préparatoires à la rentrée. La procédure y était identifiée, les membres du personnel modélisaient ce qu'ils voulaient voir apparaître comme gestes, et ce, dans les lieux et le contexte réel où les jeunes évoluent. Par exemple, on pouvait voir des enseignants en rang à deux mètres de distance se lavant tour à tour les mains avant de sortir dans la cour pour la récréation et refaire le même exercice à la fin de celle-ci. Comme il est préférable d'afficher les attentes comportementales pour les avoir bien à vue, Sandra partage une idée intéressante : "Les élèves de l'école se sont inspirés du concept d'avatar (Bitmoji) pour dessiner ${ }^{5}$ le leur en train de respecter les nouvelles mesures et ainsi passer des messages ou expliquer les nouvelles règles de vie dans toute l'école. C'était un beau projet rassembleur ! ». Dans les classes, chaque enseignant affichait les nouvelles règles illustrées en vigueur relativement à la pandémie. Des démonstrations du lavage des mains efficace ${ }^{6}$ et de l'étiquette respiratoire ${ }^{7}$ étaient réalisées devant les élèves.

\section{Le développement de relations sociales positives en présence et à distance}

La période pandémique entraîne son lot d'incertitude et d'inquiétude. Aux dires des quatre enseignants interrogés, son annonce a fragilisé le lien significatif créé depuis le début de l'année scolaire. Stéphanie déclare : «Il fallait trouver rapidement un moyen de rendre possibles les relations interpersonnelles avec les élèves, entre élèves et avec les parents. Tout le monde vivait du stress et des changements imposés. Il fallait être là l'un pour l'autre».

Dans leur façon de penser, l'enseignement à distance, les quatre enseignants ont d'emblée accordé une importance aux relations. Les enseignants ont d'abord téléphoné aux élèves à la maison pour prendre des nouvelles de leur état affectif et émotionnel, pour les inciter à s'exprimer. Ces rencontres téléphoniques 
individuelles ont généralement été appréciées des élèves et de leurs parents. Par la suite, les premières vidéoconférences visaient essentiellement à reprendre contact, à faire parler les élèves à propos de leur vécu des dernières semaines en confinement, hors classe. C'est la qualité de la relation qui était ciblée par les échanges. Sur une note positive, Mélanie raconte : "Je me suis intéressée à ce qui est nouveau pour eux, à ce qui les rend heureux même s'ils font face à l'adversité ». Les enseignants vivaient eux aussi une fin d'année scolaire anxiogène et il leur est apparu sain de l'exprimer, sans négliger d'aborder la résilience. Ils ont voulu rester souriants et chaleureux afin d'inspirer un minimum de stabilité et de sécurité tout en modélisant des habiletés sociales et communicationnelles. Pour Tristan, il est évident que la réceptivité des élèves à l'égard des savoirs essentiels du programme de formation passe avant tout par le plaisir : "Je me suis costumé à l'écran. J'ai voulu les faire rire pour qu'ils aient envie d'être là. En milieu défavorisé, c'est crucial. Sans lien affectif, l'apprentissage n'aurait pas fonctionné. En fait, les élèves ne seraient pas venus aux rencontres virtuelles. Une mère m'a envoyé une photo de sa fille en vidéoconférence avec moi. J'ai été heureux de cet envoi témoignant de nos efforts conjugués pour maintenir le lien avec l'école ». Stéphanie abonde dans le même sens. Selon l'enseignante, il fallait attirer les élèves avant de penser à une communication pédagogique autour d'une situation éducative.

En présence, lors de la rentrée de mai, Sandra a aussi pris la décision de miser d'abord sur les relations sociales positives. Elle était consciente de toutes les ruptures temporaires et des changements associés aux derniers mois et à ceux à venir. En accord avec les membres de l'équipe-école, Sandra a convenu de faire revivre une rentrée scolaire. Via une plateforme de vidéoconférence, elle a montré le réaménagement de la classe en rangées, a parlé ouvertement de sa déception quant à l'abandon de la classe flexible et des raisons justifiées de le faire. Avec transparence, elle s'est exprimée ainsi devant les élèves : «Voici NOTRE nouvelle classe. La voilà ! Il est certain que je préférais notre classe flexible, mais il faut ce qu'il faut pour qu'on se protège tous. Nous allons tranquillement nous adapter. Sachez que ceux qui resteront à la maison seront aussi avec nous en vidéoconférence. Nous pourrons nous voir et nous écouter régulièrement. Vous serez physiquement dans la classe ou à l'écran dans la classe ». Le jour J de cette deuxième rentrée, il y avait des ballons dans la classe. Un doux message d'accueil incluant l'emoji personnalisé de l'enseignante aux couleurs de l'arc-en-ciel figurait au tableau numérique interactif. Les élèves et l'enseignante ont fait une causerie visant à parler du confinement, de la rentrée post-confinement et des changements entraînés par la crise sanitaire. Sandra raconte : « La causerie avait un ton positif, en concordance avec le mouvement \#çavabienaller visant à mettre un peu de bonheur et de résilience dans ce quotidien chamboulé. En revanche, ils ont eu la tribune pour exprimer déception, tristesse, colère ». Sensible à l'importance d'accueillir toute la gamme d'émotions, Sandra a invité une professeure en gestion de classe pour animer une activité extérieure concernant la régulation émotionnelle et les comportements prosociaux. Sandra mentionne : "Le contexte faisait en sorte qu'il était impératif d'aborder les émotions ainsi que les différentes intensités de celles-ci. À l'aide de la technique d'impact du verre d'eau qui peut déborder, la professeure a abordé la gestion des émotions. Par la suite, les élèves devaient acter quelques mises en situation en nommant les émotions suscitées, en situant leur intensité et les comportements prosociaux à adopter. C'est une professeure avec qui je collabore. J'ai animé un atelier auprès de ses étudiants en éducation préscolaire et enseignement primaire. Elle a fait de même auprès de mes élèves ». Lenseignante encourage ces initiatives de collaboration interinstitutionnelle au service des élèves. 


\section{L'adaptation de stratégies pour capter l'attention et maintenir l'engagement des élèves}

La motivation des élèves à apprendre a été fragilisée par le contexte pandémique. Qu'est-ce qui a pu pousser des élèves du préscolaire primaire à s'engager dans leurs apprentissages lorsque leur école était fermée, qu'ils sont devenus malgré eux des apprenants à distance face à des contenus dits facultatifs ? Selon les quatre enseignants, il fallait agir en fonction d'une étroite collaboration école-famille pour influencer positivement les élèves à s'engager.

À distance, la motivation ne dépendait pas seulement de l'attitude et des perceptions des élèves, du climat ainsi que de l'attitude et des pratiques des enseignants. Les parents, peu importe leurs ressources, étaient notamment très sollicités puisque les élèves avaient des besoins non négligeables de stimulation intellectuelle. Les trousses pédagogiques du MÉES envoyées aux parents avaient pour but de consolider les acquis et le goût d'apprendre durant la période de fermeture des écoles. L'envoi hebdomadaire des trousses portait la mention «optionnel » ou "facultatif ». À cet égard, Stéphanie affirme avoir dû ramer à contre-courant : "Je sais bien qu'il fallait enlever de la pression sur les épaules des parents qui n'ont pas forcément le temps, les compétences pour encadrer des apprentissages. J'ai eu à faire de multiples tutoriels vidéo pour aider les parents à développer des compétences technologiques. La motivation et la participation des élèves passaient entre autres par cela. Aussi, il fallait dire que c'est facultatif, dire que le contenu des trousses n'allait faire l'objet d'aucune évaluation. On ne voulait pas creuser les inégalités. Si certains élèves ont pris plaisir à s'engager dans la découverte des contenus proposés, je vous assure que ce n'est pas le cas de la majorité de mes élèves. Je suis devenue encore plus qu'avant une agente de motivation auprès d'eux et de leurs parents. J'ai volontairement insisté sur le caractère fort recommandé de la consolidation des acquis. J'ai initié des projets à remettre qui étaient à la portée de tous. Ils déposaient les travaux et je fournissais de la rétroaction écrite, orale et vidéo à l'aide d'outils comme Loom et Padlet ». Sandra, quant à elle, a suscité l'engagement des élèves à l'aide de projets d'écriture variés et significatifs : "Les élèves devaient écrire un texte selon une intention différente toutes les semaines. Ils me l'envoyaient par courriel ou par Seesaw. Cela me permettait de garder un contact direct avec chacun, car je leur offrais une rétroaction pouvant ressembler à une petite correspondance entre nous. Sur Zoom, les élèves partageaient leur texte en les lisant aux autres ». Tristan se remémore le chemin parcouru depuis l'animation de ses premières vidéoconférences : « Au début, c'est certain que j'ai senti que les enfants ne venaient pas pour apprendre, mais pour voir le monde. À un moment donné, se regarder le blanc des yeux, c'est long et ennuyeux. Tranquillement, j'ai commencé à faire des joggings en français et en mathématiques ou à leur poser des questions plus précises. Une fois ça mis en place, nous sommes rentrés dans des leçons en ligne plus dirigées ». Pour Tristan, il fallait regagner la motivation des élèves de manière progressive, un pas à la fois. Même si le contexte n'était pas favorable à la motivation, les enseignants ont encouragé les élèves et leur famille à déployer des efforts en les soutenant de manière synchrone ou asynchrone, et ce, sur les plans pédagogique et technologique.

En classe, dès le mois de mai, Sandra enseignait à la fois en présence et à distance en ouvrant fréquemment sa plateforme de vidéoconférence. Les élèves à la maison recevaient encore les trousses pédagogiques bonifiées en fonction de la planification pour les élèves en présentiel. Les journées commençaient par de l'analyse grammaticale. Entre les périodes d'apprentissage et le respect de la distance physique recommandée, l'enseignante animait des pauses actives. Elle exploitait aussi grandement le chariot de 
tablettes afin de proposer des évaluations formatives sans contact à l'aide de questionnaires en ligne. Sandra déclare : «Les élèves ont adoré les questionnaires Kahoot. Je leur ai demandé d'être attentifs à certains modes plus transmissifs, mais je combinais avec des leçons ludiques et un usage d'applications numériques, dont la Constellation de l'ours, un coup de cœur pour certains élèves en difficulté et leurs parents ». L'enseignante a eu à faire des efforts pour rendre la consolidation des acquis intéressante. Les stratégies adoptées ont été variées et différenciées. Les quatre enseignants soutiennent que la rétroaction écrite et orale personnalisée et fréquente sur les travaux demandés aux élèves était plus que nécessaire, bien que chronophage. Par le biais de communications fréquentes entre l'école et la maison et d'une collaboration avec les parents, les enseignants, experts de la motivation et de la réussite scolaire, suscitaient l'engagement.

\section{La gestion éducative des comportements d'indiscipline}

Avec l'arrivée de la crise sanitaire due à la pandémie de la COVID-19, les enseignants ont établi de nouvelles attentes. Il aurait été utopique de croire qu'aucun élève n'allait transgresser les règles. Lors de chaque rencontre à distance ou en présentiel, les enseignants s'assuraient de faire respecter ces règles. Selon une stratégie préventive, et en fonction du principe des cinq «C » (Gaudreau, 2017), ils ont eu à revenir, encore et encore, à un enseignement explicite des règles et des comportements attendus. Les consignes devaient être claires, concrètes, appliquées avec constance et cohérence et suivies de rétroactions conséquentes de la part de l'enseignant. De fait, les règles relatives à la nétiquette ou aux mesures de la DSP, par exemple, devaient être formulées clairement en tenant compte de l'âge des élèves et être concrétisées dans des comportements observables. Au regard de la constance, les règles devaient être maintenues et appliquées par tous. Avec cohérence, les enseignants donnaient l'exemple dans leurs gestes et leurs paroles.

À distance, Mélanie priorisait la routine, le rappel des consignes et la modélisation pendant les vidéoconférences. Elle mentionne : «Donner l'exemple me permettait d'indiquer à ceux qui parlaient à tout moment de porter attention au respect du droit de parole pour éviter le chao et patienter jusqu'à la causerie. C'est si précieux quand on est sur Zoom avec des enfants de cinq-six ans qui ont beaucoup à dire ». Les options d'animation des différents outils de vidéoconférence donnaient aux enseignants la possibilité de fermer le micro de certains élèves lorsque les bruits environnants nuisaient à la concentration. Les enseignants affirment à l'unisson qu'il n'était pas question d'abuser de cette option. Stéphanie en parle ainsi : «À l'occasion, je pouvais dire à un élève que je coupais son micro le temps qu'il se dirige vers un endroit plus tranquille. À la maison, il était essentiel de créer un espace rendant possible la concentration ».

À la mi-mai, lors du retour en classe, Sandra soulève le défi de fournir une aide aux élèves indisciplinés sans trop de proximité, avec une visière et un masque : "J'avais l'habitude de marcher ma classe flexible, d'exploiter la proximité pour intervenir quand j'anticipais des inconduites. Je ne pouvais pas exploiter la proximité ni le toucher comme avant. En revanche, je pouvais établir un contact visuel, me servir de gestes aussi. Même avec un masque, quand j'arrêtais de parler, que je fronçais les sourcils et que je pointais une règle de classe affichée, les élèves savaient quoi faire. Je devais faire parler mes yeux. Quand je faisais les ailes d'oiseau, les élèves comprenaient qu'ils devaient se reculer l'un de l'autre pour respecter la distance ». L'enseignante et les membres de l'équipe-école avaient convenu de certains 
signes non verbaux pour rappeler la distanciation physique et le lavage des mains, notamment. Ils ont également décidé, en concertation, de restreindre les interdits en limitant l'accès au matériel trop difficile à désinfecter. Lorsque des élèves transgressaient des règles, des rappels par des directives alpha leur étaient faits et ils étaient responsabilisés en leur faisant vivre les conséquences liées à leurs choix : retourner se laver les mains, reprendre une entrée dans l'école selon le sens de la circulation. La plupart du temps, les élèves recevaient de la rétroaction positive lorsqu'ils répondaient aux attentes à leur égard. Il pouvait s'agir de remerciements et de félicitations, de messages écrits s'adressant à tous sur le réseau social de l'école, sur la plateforme numérique de classe, par courriel ou par clavardage pour s'adresser plus particulièrement à un élève et ses parents. Il est arrivé à Sandra de contacter des parents après avoir fait une analyse d'un comportement inadéquat. Dans ce contexte, les échanges collaboratifs visaient à trouver des solutions et parfois à ajuster le plan d'intervention. Pandémie ou pas, les élèves à besoins particuliers étaient considérés.

\section{Conclusion}

Qu'elle soit à distance ou en présence, la reprise de l'école était certes complexe. Cette reprise était possible et bénéfique, bien qu'elle ait nécessité adaptabilité et flexibilité de par les défis qu’elle a engendrés. La scolarisation des élèves ne pouvait être viable sans les enseignants et leurs collaborateurs. L'adaptation de la gestion de la classe au contexte de pandémie a pu permettre aux élèves de reprendre leurs apprentissages, et ce, avec toute une organisation réfléchie en fonction des différentes directives.

Les enseignants qui ont accepté de faire part de leur expérience en cette fin d'année scolaire 2020 bien particulière offrent aux acteurs éducatifs des pistes d'exploration parmi d'autres pour mieux repenser la gestion de classe et l'adapter à la crise actuelle. Par les stratégies qu'ils ont déployées à distance ou en présence, ils ont donné aux élèves la possibilité de briser leur isolement en partageant leur vécu et leurs émotions, mais aussi en réalisant des apprentissages. Les enseignants leur ont redonné une voix, une place et un certain pouvoir-agir sur leur avenir, malgré les chambardements. Qu'adviendra-t-il de la prochaine rentrée ? Qu'adviendra-t-il de la prochaine année et de celles qui suivront? Les réponses sont partielles, mouvantes et l'incertitude plane toujours.

La crise actuelle est encore bien présente et nécessitera de concilier les conditions de retour en classe avec la réalité de chaque milieu scolaire, les besoins des élèves ainsi qu'en tenant compte des directives des différents ministères. Pour le présent et l'avenir de leurs élèves, les enseignants et leurs collaborateurs devront s'unir avec inventivité pour relever les différents défis d'organisation physique et pédagogique. Les solutions visant la santé globale et les valeurs inclusives de l'école devraient être planifiées dans une démarche collaborative empreinte d'authenticité, de confiance, d'autodétermination et être orientées vers des objectifs collectifs et des décisions claires prises en concertation. 


\section{Notes}

1 https://enclasse.telequebec.tv/contenu/aux-toilettes/1460

2 http://elisegravel.com

3 https://recitfad.ca/netiquette-pour-lapprenant-en-visioconference/

4 https://www.facebook.com/ecoleduruisseau/ (les photos et vidéos peuvent être retirées à tout moment par l'administrateur)

5 https://www.facebook.com/ecoleduruisseau/ (les photos et vidéos peuvent être retirées à tout moment par l'administrateur)

6 https://zonevideo.telequebec.tv/media/54135/le-lavage-des-mains/l-ecole-a-la-maison

7 https://fipeq.org/ressources/covid-19-coronavirus/

\section{Références bibliographiques}

Caron, J. (2012). Quand revient septembre : guide sur la gestion de classe participative, $2^{\mathrm{e}}$ éd. Chenelière Éducation.

Caron, J., Portelance, L. et Marzouk, A. (2020, 13 février). Acculturation scientifique dans une visée de développement de la compétence professionnelle des stagiaires en matière de gestion de classe [communication orale]. $6^{\mathrm{e}}$ colloque du gEvaPP. Fribourg, Suisse.

Connac, S. (2020). Une pédagogie coopérative sans contact? Cahiers pédagogiques. https://www.cahiers-pedagogiques.com/Une-pedagogie-cooperative-sans-contact

Duchesne, C. et Kane, R. (2010). Défis de l'insertion professionnelle et dispositifs d'encadrement. McGill Journal of Education, 45(1), 63-80. https://doi.org/10.7202/1000030ar

Dufour, F., Portelance, L., Pellerin, G. et Boies, I. (2019). La préparation à l'insertion dans la profession : regard d'enseignants débutants sur la formation initiale au Québec. Éducation et Formation, e-315, 29-45.

Gaudreau, N. (2017). Gérer efficacement sa classe. Les cinq ingrédients essentiels. Presses de l'Université du Québec.

Girouard-Gagné, M. et Paré, M. (2015). Différencier les pratiques pédagogiques pour tenir compte de l'hétérogénéité : une question de compétence en gestion de classe? Revue canadienne des jeunes chercheur(e)s en éducation, 999(999). 10-19.

Harvey, C. (à paraître). Conception et mise à l'essai d'un dispositif de codéveloppement professionnel en gestion de classe auprès d'enseignants débutants du secondaire en adaptation scolaire et sociale [mémoire de maitrise inédit]. Université du Québec à Rimouski.

Leroux, M. et Paré, M. (2016). Mieux répondre aux besoins diversifiés de tous les élèves. Des pistes pour différencier, adapter et modifier son enseignement. Chenelière Éducation.

Martin, B., Pidoux, M., et Brülhart, E. (2019, 25 avril). Apports et manques dans la formation à la gestion de classe [communication orale]. 6ème colloque international en éducation: enjeux actuels et futurs de l'éducation de la formation et de la profession enseignante, Montréal, QC, Canada.

Martineau, S. et Vallerand, A.-C. (2007). Les dispositifs pour soutenir l'insertion professionnelle des enseignants. http://www.cnipe.ca/IMG/pdf/Martineau-Vallerand-2007-Les-dispositifs---.pdf

Rojo, S. et Bergeron, G. (dir.) (2017). L’intervention psychosociale par la nature et l'aventure: fondements, processus et pistes d'action. Presses de l'Université du Québec.

Savoie-Zajc, L. (2019). Les pratiques des chercheurs liées au soutien de la rigueur dans leur recherche : une analyse d'articles de Recherches qualitatives parus entre 2010 et 2017. Recherches qualitatives, 38(1), 32-52. https://doi.org/10.7202/1059646ar

\section{Pour citer cet article}

Caron, J., Gaudreau, N., Harvey,C., Sicard, S., Robitaille, S., Arbour, M. et Brochu, T. (2020). La gestion de classe au primaire en contexte de pandémie. Formation et profession. 28 (4 hors-série). 1-12. http://dx.doi.org/10.18162/fp.2020.674 